Trabajo seleccionado para su publicación por el jurado del Premio Estudios Financieros, formado por: don Antonio Bautista García-Vera; don Luis Alberto Guijarro Rojo; doña Ana Hidalgo Tena; doña Blanca Lozano Cutanda y don José Eugenio Martínez Falero.

Virginia Jiménez Rodríguez', Carmen Llopis Pablos², Patricia Jara Calaforra Faubel ${ }^{3}$, Pierre Josué Almagro Lapo ${ }^{4}$, Jesús María Alvarado Izquierdo ${ }^{5}$ y Aníbal Puente Ferreras ${ }^{6}$

\title{
Utilización de códigos QR para la evaluación continua en educación superior
}

\section{Extracto:}

La implementación de las tecnologías de la información y la comunicación (TIC) en el ámbito educativo se ha acelerado en los últimos años. En el entorno universitario se utilizan plataformas educativas que dan acceso a los contenidos de diferentes asignaturas, así como la comunicación on-line entre docente y alumnado.

El presente proyecto pretendía mejorar la calidad docente, así como la motivación y satisfacción de los estudiantes del primer curso de grado, a través de la inserción de nuevas herramientas TIC [formularios Google y códigos QR (quick response codes)] que permitieran al alumnado la evaluación continua de su propio aprendizaje, haciendo especial hincapié en la aplicación de estrategias metacognitivas para la resolución de problemas.

Se realizó durante el curso académico 2014-2015 en la asignatura de Psicología Básica (prácticas). La asignatura Psicología Básica se imparte en el primer curso del grado de Trabajo Social en la Universidad Complutense de Madrid. Consta de seis créditos ECTS (Sistema Europeo de Transferencia y Acumulación de Créditos) y, como tal, los estudiantes reciben dos horas de clase teórica y una hora de clase práctica a la semana. Fue durante la hora semanal de prácticas donde se llevó a cabo el presente proyecto de innovación.

Fecha de entrada: 03-05-2016

Fecha de aceptación: 12-07-2016

Palabras claves: códigos QR (quick response code), tecnologías de la información y la comunicación (TIC), evaluación continua, educación superior, Trabajo Social, estrategias metacognitivas.

\footnotetext{
${ }^{1}$ V. Jiménez Rodríguez, profesora asociada del Departamento de Psicología Básica Il de la Facultad de Trabajo Social de la Universidad Complutense de Madrid.

${ }^{2}$ C. Llopis Pablos, doctoranda de la Facultad de Psicología de la Universidad Complutense de Madrid.

${ }^{3}$ P. J. Calaforra Faubel, doctoranda de la Facultad de Psicología de la Universidad Complutense de Madrid.

${ }^{4}$ P. J. Almagro Lapo, graduado en Psicología en la Facultad de Psicología de la Universidad Complutense de Madrid.

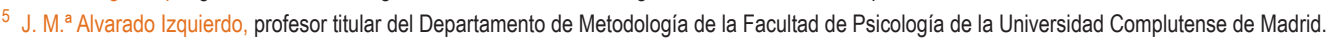

${ }^{6}$ A. Puente Ferreras, catedrático de Psicología del Departamento de Psicología Básica Il de la Facultad de Trabajo Social de la Universidad Complutense de Madrid.
}

Este proyecto ha sido realizado gracias a la financiación de los Proyectos de Innovación y Mejora de la Calidad Docente (PIMCD) de la Universidad Complutense de Madrid (proyecto núm. 335) y de la Facultad de Trabajo Social de la Universidad Complutense de Madrid durante el año 2014. 


\section{Using QR codes for}

\section{continuous}

\section{assessment}

\section{in higher education}

\section{Abstract:}

The implementation of information and communications technology (ICT) in education has accelerated in recent years. At university level educational platforms that provide access to the contents of different subjects are used, as well as on-line communication between teachers and students.

This project intended to improve teacher quality and motivation and satisfaction in 1st grade students, through the insertion of new ICT tools [forms Google and QR codes (quick response codes)] that allow students the continuous assessment of their own learning, with particular emphasis on the application of metacognitive strategies for problem solving.

It was conducted during the academic year 2014-2015 in the subject of Basic Psychology (practices). The subject Basic Psychology is taught in 1st Degree of Social Work at the Complutense University of Madrid. It consists of six ECTS (European Credit Transfer and Accumulation System) credits and as such, students receive two hours of lecture and practical class one hour each week. It was during the weekly hour of practice which was carried out this innovation project.

Keywords: QR codes (quick response codes), information and communications technology (ICT), continuous assessment, degree, Social Work, metacognitive strategies.

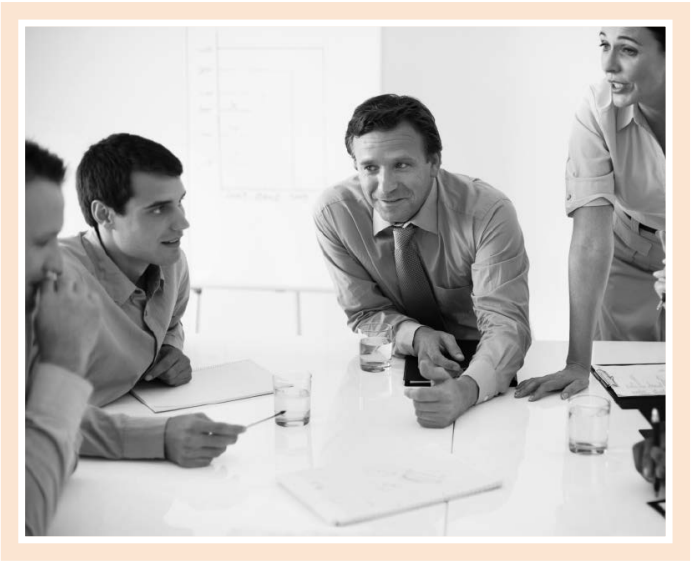

\section{INTRODUCCIÓN}

\subsection{Evaluación continua en la educación su- perior}

Cuando nos referimos al concepto de «evaluación» hacemos alusión a la valoración de la calidad del aprendizaje adquirido por los estudiantes.

Tradicionalmente, el modelo de evaluación que se ha utilizado, y que se sigue usando, es el que está formado por los siguientes elementos: recogida de información, aplicación de los criterios de calidad y emisión de un juicio sobre la calidad del aprendizaje. Todo esto lo realiza el docente. Él es quien elabora los criterios de evaluación y quien decide qué calificación obtiene el alumno en la materia evaluada.

Con la implantación de los cambios y las reformas europeas, principalmente en el sistema de créditos y en los papeles de los alumnos y docentes, el modelo de evaluación también está cambiando. Con este nuevo marco, el aprendizaje y la evaluación giran en torno al estudiante, y aunque el profesor mantiene su rol de evaluador, lo hace de forma diferente al modelo tradicional.

Debido a estos cambios, es imprescindible revisar el proceso evaluativo en el ámbito universitario puesto que de él sigue dependiendo si la valoración del alumno en la consecución de los objetivos es positiva o negativa. Por tanto, debe ser una evaluación que valore, adecuadamente, el esfuerzo y el trabajo realizados por parte del estudiante; $y$ «debe tener 
una sistemática similar al proceso de aprendizaje que ha realizado el estudiante» (Delgado et al., 2005).

La evaluación ha pasado de ser la tarea obligatoria que se realizaba al finalizar los trimestres, cursos, e independiente de las actividades formativas, a considerarse un proceso integrado e interconectado con el proceso de formación.

Si nos remontamos a la primera definición de «evaluación» que se conoce, hacemos referencia a Ralph W. Tyler (citado en Delgado et al., 2005), que ya en 1942 definió este concepto como «el proceso que tiene por finalidad valorar en qué medida se han conseguido los objetivos que se habian previsto» 0 , en otros términos, «valorar el cambio ocurrido como efecto de la formación».

Mucho tiempo ha pasado desde que Tyler diera a conocer su definición, y actualmente no se tiene en cuenta, puesto que han sido muchos los cambios en la enseñanza.

En este tiempo se plantea más como el proceso, desde el inicio de la formación hasta el final de ella, que pretende descubrir tanto aquellos componentes que están funcionando adecuadamente como los que no funcionan, con el fin último de asegurar una calidad del proceso de formación y aprendizaje. Esto es, una evaluación basada más en el proceso, en la adquisición de competencias y habilidades, y no basada en el producto de aprendizaje en los diferentes entornos.

Dependiendo de los objetivos que se establezcan en el proceso de aprendizaje (objetivos en cuanto a conocimientos, habilidades, hábitos y actitudes en el estudiante), se pueden diferenciar diversos modelos de evaluación. Por ello, teniendo en cuenta la finalidad y el momento en que se va a realizar, pueden diferenciarse los siguientes modelos:

- Evaluación diagnóstica o inicial.

- Evaluación formativa.

- Evaluación progresiva o continua.

- Evaluación sumativa o final.

\section{Con este tipo de evaluación progresiva o continua, el profesor es capaz de realizar un mayor seguimiento del trabajo que hacen los estudiantes y su evolución. Es una evaluación más justa y más ajustada a los conocimientos que ha adquirido el estudiante}

Además, en los últimos años ha aparecido un nuevo enfoque que propone otro modelo de evaluación: la evaluación alternativa, que pretende observar y valorar más directamente el trabajo realizado por los estudiantes. Así, el profesor no deja de ser el evaluador, pero ya no es el único elemento de este control, sino que ahora comparte esta tarea con los estudiantes, que pasan a desempeñar un rol muy importante en su propia evaluación y/o en la de sus compañeros. Esta nueva forma de evaluar es un modelo de evaluación formativa que requiere una evaluación continuada.

Si tenemos en cuenta el sistema tradicional de evaluación final al que los estudiantes orientaban todo su esfuerzo, y que estaba dirigido fundamentalmente a aprobar los exámenes (o trabajos) para los que se les facilitaban unos contenidos, es fácil apreciar que no basta con valorar, al finalizar el proceso de aprendizaje, la asimilación o no de los contenidos, sino que los docentes deben, además, proporcionar tareas, recursos evaluables que contribuyan a la asimilación, al desarrollo y a la integración de los contenidos y competencias previstas (learning by doing).

Con este tipo de evaluación progresiva o continua, el profesor es capaz de realizar un mayor seguimiento del trabajo que hacen los estudiantes y su evolución. Es una evaluación más justa y más ajustada a los conocimientos que ha adquirido el estudiante.

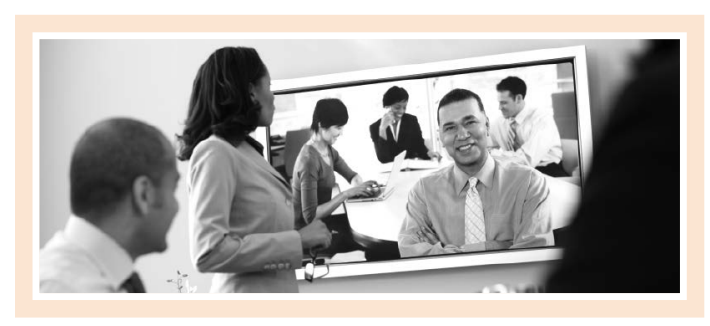


Este modelo de evaluación continua ofrece una serie de ventajas tanto para los propios estudiantes como para los docentes. Los primeros, al ser partícipes y conocedores en todo momento de su propio proceso de evaluación, tienen más probabilidades de alcanzar el éxito en estas materias. Además, como obtienen los resultados de las actividades que van realizando de forma rápida (feedback), tienen la posibilidad de ir modificando, reorientando su trabajo, si es necesario, y ser mucho más activos en el aula.

El docente también se beneficia de una serie de ventajas a la hora de poder mejorar y reorientar su papel como informador/orientador de los estudiantes; también puede mejorar la metodología que utiliza en las clases y llegar al objetivo último que es la valoración del esfuerzo, del rendimiento y de la adquisición de conocimientos, estrategias y habilidades por parte de los estudiantes a lo largo de la duración de la asignatura.

¿Y cómo debe ser el diseño de esta evaluación continua? Debe estar centrada en tres elementos fundamentales:

- Planificación adecuada de la materia que se va a impartir.

- Información al estudiante de los objetivos propuestos, de los criterios de evaluación, de los recursos, de las actividades, del calendario, etc. El alumno debe ser conocedor de todo ello, principalmente de los objetivos de la asignatura y de los criterios de valoración, puesto que va a ser evaluado teniéndolos en cuenta.

- Actividades concretas de evaluación de los diferentes temas, criterios concretos de corrección, etc., que deben ser coherentes con el proceso de aprendizaje y con la metodología que se ha seguido durante el curso.

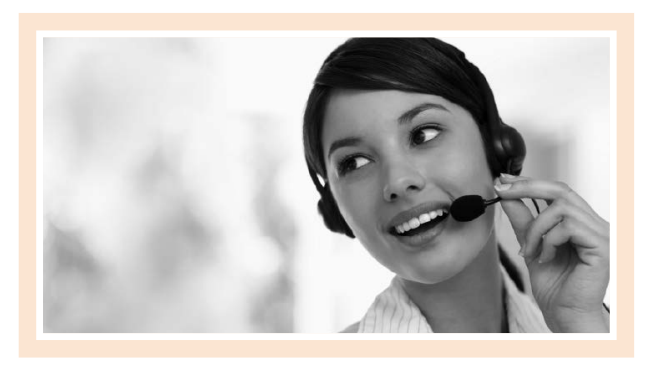

\section{A pesar de las ventajas que parece tener este modelo de evaluación continua en la enseñanza, más aún en la enseñanza superior, el grado de implementación varía sustancialmente en los diferentes entornos (presenciales y virtuales)}

La evaluación continua debería llevarse a cabo en todas las materias. Es importante explicar claramente a los estudiantes, al iniciar una asignatura, en qué consiste este tipo de evaluación continua. Con este modelo se trata de plantear diversas actividades que serán evaluadas y que tendrán que ir realizando durante el curso con una doble finalidad: planificar su propio ritmo de trabajo y evaluar el proceso de aprendizaje para conseguir el objetivo final, que es superar la materia habiendo adquirido las estrategias, las habilidades y los conocimientos requeridos.

Este modelo de evaluación continua supone para el profesor una carga adicional de trabajo que puede disminuir con un diseño de evaluación automatizada (plantillas con las respuestas de las actividades) y/o implementando las TIC y software especializados.

Según las experiencias de los últimos años, las principales ventajas que se obtienen con la evaluación continua en la educación superior son una mayor motivación (generalmente bastante descuidada a pesar de su relevancia para el aprendizaje); mayor interacción profesor-alumno; incremento de la orientación, acompañamiento del estudiante en su proceso de aprendizaje por parte del docente; y la valoración positiva, por parte de los estudiantes, de la dedicación del profesor en su materia dentro de este sistema de evaluación.

A pesar de las ventajas que parece tener este modelo de evaluación continua en la enseñanza, más aún en la enseñanza superior, el grado de implementación varía sustancialmente en los diferentes entornos (presenciales y virtuales).

En los entornos virtuales, la puesta en marcha de la evaluación continua en el nuevo marco europeo es 
completa y funciona correctamente con la metodología y las ventajas que se han expuesto anteriormente: todas las actividades son evaluables; se obtienen los resultados de las tareas inmediatamente después de haberlas realizado y antes de iniciar las siguientes; hay una mayor interacción-comunicación entre el profesor y el estudiante; estos entornos facilitan mucho más el intercambio de comunicación de forma simultánea (entrega de actividades, dudas, inquietudes, etc.) y una mayor organización del trabajo personal.

Por el contrario, en los entornos presenciales sigue existiendo una resistencia a la adopción de este modelo de evaluación. En la mayoría de los casos siguen con los modelos tradicionales y no todas las actividades son evaluables, sino que se centran en casos prácticos difícilmente evaluables con objetividad. Además, las correcciones de estos no son inmediatas y la interacción-comunicación entre docente y estudiante es mucho menor.

Por ello, parece ser que los docentes que imparten sus clases en entornos presenciales son los que deben hacer un verdadero esfuerzo en la implementación de la evaluación continua para adaptarse a las nuevas necesidades con la introducción de las TIC en el Espacio Europeo de Educación Superior (EEES).

\subsection{Metacognición en la educación superior}

En los últimos años, la universidad está siendo instada a realizar multitud de cambios, sobre todo metodológicos, debido a las directrices marcadas por el EEES. Continuamente se está cuestionando si estos cambios serán positivos, viables en la enseñanza, porque parece ser que poco se ha transfor-

\section{(...) los docentes que imparten sus clases en entornos presenciales son los que deben hacer un verdadero esfuerzo en la implementación de la evaluación continua para adaptarse a las nuevas necesidades con la introducción de las TIC}

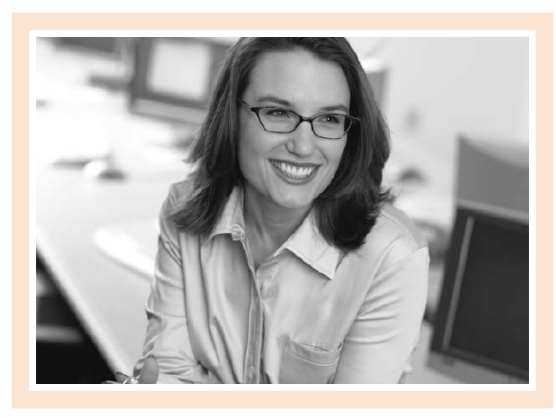

mado el sistema en nuestras aulas, y, en algunas universidades en las que se han introducido y se llevan a cabo estos cambios en su totalidad, son tan lentos que casi ni se aprecian en la formación y el desarrollo de los estudiantes y profesores.

Pero realmente estos cambios van más allá de los meramente burocráticos, de tiempos, horarios, etc. Se trata de una nueva filosofía educativa en la que el objetivo último de la enseñanza no es, únicamente, la trasmisión de conocimientos del profesor hacia el alumno, sino «hacerles competentes en el uso de los ya adquiridos» (Pozo y Monereo, 2009).

Uno de los cambios más importantes son los nuevos roles del profesor y del alumno. La enseñanza superior debería enseñar a los estudiantes a ser partícipes de su propio proceso de aprendizaje, a construir su propio conocimiento (adquisición de un rol más activo y autónomo), en lugar de ser simples receptores de los contenidos que les son transmitidos por el profesorado. El docente se convierte en orientador y dinamizador del aprendizaje del alumnado, y este, en generador de su propio aprendizaje. Por lo que una meta primordial de la formación universitaria debería ser preparar a los alumnos para que sean capaces de organizar la información que reciben para transformarla en verdadero conocimiento. Esto es, «aprender a aprender». Este nuevo escenario hace mayor hincapié en lo que aprende el alumno que en lo que enseña el docente, por lo que parece claro apostar por una metodología de tipo más constructivista. La enseñanza se construye sobre lo ya aprendido 


\section{La enseñanza superior debería enseñar a los estudiantes a ser partícipes de su propio proceso de aprendizaje, a construir su propio conocimiento (...), en lugar de ser simples receptores de los contenidos que les son transmitidos por el profesorado}

(conocimientos previos) y se da una interconexión entre los nuevos contenidos (ampliando los esquemas de conocimiento del aprendiz), la guía del que enseña (docente) y la actividad cognitiva de quien construye el nuevo conocimiento (el propio estudiante). Todo ello alude al conocimiento que tiene la persona sobre sus propios procesos y productos de pensamiento, incluyendo también cualquier información relevante en el proceso de aprender (Flavell, 1976); esto es, la metacognición. Algunos investigadores de esta área la consideran el «centro de control» del sistema cognitivo (Flavell, 1971, 1981 y 1987; Flavell y Wellman, 1977; Schraw, 1998). A este respecto, Brown (1980 y 1987) ofrece una descripción precisa que incluye dos dimensiones relacionadas: el conocimiento y la regulación de la cognición. La primera alude al qué sabemos de ella, y la segunda, al cómo la regulamos; por lo que estaríamos hablando de la autorregulación como clave del proceso de aprendizaje. Pero el aprendizaje autorregulado no solo depende de factores cognitivos, sino también de factores motivacionales (Burón, 1996; Mateos, 2001; Pintrich y De Groot, 1990). Si los factores cognitivos se relacionan con la competencia para llevar a cabo una tarea, los factores de motivación se asocian con la actuación o el rendimiento en la tarea (Jiménez et al., 2009). Así, la relevancia que tiene en el aprendizaje el proceso de autorregulación resulta obvia, y, sin embargo, diversos estudios revelan que los estudiantes de todas las edades «tienen dificultades para desplegar sus habilidades metacognitivas, las cuales juegan un rol fundamental en el proceso autorregulatorio cuando el aprendizaje se lleva a cabo en contextos abiertos» (Suárez et al., 2012).
Con todo, parece ser que «aprender a aprender» se establece como una de las demandas esenciales que debe satisfacer la formación universitaria. Además, debe facilitar que los estudiantes puedan adquirir las estrategias y competencias que les dirijan a acometer con éxito su formación y desarrollo personal, académico y profesional, generalizando sus propios aprendizajes.

El objetivo pasa a ser formar a profesionales capaces de utilizar y gestionar de forma autónoma el conocimiento, cuya finalidad sea generar soluciones a nuevos problemas que se planteen; es decir, formar personas reflexivas para conseguir un aprendizaje eficaz. Pero un aprendizaje eficaz requiere de una gestión metacognitiva, que supone el conocimiento y el control del propio pensamiento, llegando a una autorregulación del aprendizaje.

En este tipo de aprendizaje se constituyen dos aspectos muy relacionados:

- El conocimiento que las personas desarrollamos sobre nuestro propio conocimiento (intereses, capacidades, experiencias, habilidades).

- El control que tenemos sobre cómo usamos nuestro propio conocimiento al enfrentarnos a situaciones y problemas concretos.

Este llamado «metaconocimiento» se desarrolla a lo largo de tres procesos diferentes que ocurren simultáneamente cuando realizamos nuestras tareas:

- Planificación.

- Supervisión.

- Proceso de evaluación.

La planificación tiene que ver con activar conocimientos previos, plantear objetivos, seleccionar estrategias, definir un plan de acción, etc. La supervisión consiste en determinar la efectividad de las estrategias de solución de la tarea, descubrir errores y, en función de ellos, reorientar las acciones seleccionando otras estrategias. La evaluación alude a establecer la correspondencia entre los objetivos propuestos y las metas logradas, decidir sobre la mejor solución y apreciar la validez y la pertinencia de las estrategias aplicadas (Ríos, 1991). Así, se puede establecer que la metacognición consiste en tres procesos (Jiménez, 2004) (véase figura 1). 
Figura 1. Procesos metacognitivos

$\left.\begin{array}{l}\text { Planificación: } \\ \text { - Desarrollar un plan de acción }\end{array} \quad \begin{array}{l}\text { Supervisión: } \\ \text { Supervisar el plan de acción }\end{array}\right) \begin{aligned} & \text { Evaluación: } \\ & - \text { Evaluar el plan }\end{aligned}$

Fuente: extraído de Jiménez (2004)

Esta nueva forma de aprender y construir el conocimiento no es suficiente para formar alumnos reflexivos, críticos, capaces de construir su conocimiento y resolver los problemas que se les planteen. Esta habilidad es aprendida y, para ello, se necesitan docentes que les muestren cómo realizar este tipo de trabajo; que les enseñen estrategias, herramientas, cómo integrarlas para conseguir las metas propuestas y asi llegar a un aprendizaje autorregulado y, por ende, autónomo.

\section{Estas herramientas tecnológicas promueven entornos, estrategias$$
\text { y herramientas de }
$$$$
\text { aprendizaje con el }
$$$$
\text { objetivo de incrementar }
$$$$
\text { el interés y la }
$$$$
\text { motivación por parte }
$$$$
\text { del alumnado, además }
$$$$
\text { de favorecer la labor }
$$$$
\text { docente diaria }
$$

\subsection{Metacognición y tecnologías de la informa- ción y la comunicación}

El vertiginoso avance en la organización social del conocimiento que se está dando actualmente parece estar muy vinculado al desarrollo tecnológico. En este nuevo marco, la educación superior se caracteriza por las reformas educativas europeas en las que se apuesta por la introducción de las TIC en las aulas, aprovechando el factor motivacional que tienen, así como el manejo que hace de ellas el estudiante universitario de hoy. Todo ello con el objetivo de que redunde positivamente en su propio aprendizaje. Las TIC pueden ser un medio apropiado para la potenciación y el desarrollo de las temáticas emergentes, y una herramienta muy importante en el campo de la educación. Uno de los principales objetivos de las TIC en el entorno educativo es crear individuos con mayor autonomía, facilitándoles la adquisición de recursos y conocimientos de forma organizada en diferentes entornos, aprovechando la ubicuidad de las herramientas para poder alcanzar sus objetivos.

Estas herramientas tecnológicas promueven entornos, estrategias y herramientas de aprendizaje con el objetivo de incrementar el interés y la motivación por parte del alumnado, además de favorecer la labor docente diaria.

Utilizar herramientas informáticas personales guiadas por estrategias metacognitivas (de selección, organización y elaboración, teniendo en cuenta la propia experiencia, las metas propuestas, las características personales y de la tarea que se quiere realizar) aplicadas por el alumno en beneficio de su propio aprendizaje es la base de una individualización de su espacio de aprendizaje (Zapata, 2012).

Así, al unir el constructo «metacognición» a las TIC, se podrían conocer y controlar cada uno de los pasos realizados y las he- 


\section{(...) al unir el constructo «metacognición» a las TIC, se podrían conocer y controlar cada uno de los pasos realizados y las herramientas utilizadas por parte de los estudiantes en el proceso de aprendizaje}

rramientas utilizadas por parte de los estudiantes en el proceso de aprendizaje. Además, los estudiantes podrían tener una retroalimentación de sus procedimientos en cuanto al aprendizaje, incremento en los aspectos motivacionales $y$, con ello, un aumento en la eficacia y eficiencia en su aprendizaje diario (MüIler, 2009). El docente realiza preguntas que llevan en un primer momento a la reflexión con el objetivo de llegar a la autorreflexión, teniendo muy en cuenta los aspectos motivacionales y emocionales de los estudiantes. Es un nuevo enfoque metodológico en el aula. El estudiante es protagonista y autónomo de su propio aprendizaje y el docente se convierte en dinamizador y orientador del proceso enseñanza-aprendizaje (LLopis, Jiménez y Benítez, 2015).

Una de las herramientas TIC más utilizadas es el uso del ordenador portátil en el aula, que tiene como ventaja que permite hacer más tareas con menor esfuerzo, puesto que una parte del trabajo cognitivo lo asimila la herramienta. Asimismo, esta herramienta permite implicarse en muchas tareas que de otra forma estarían fuera de su campo de acción. Son facilitadores de aprendizajes significativos, de nuevas estrategias pedagógicas, ayudan a que los alumnos

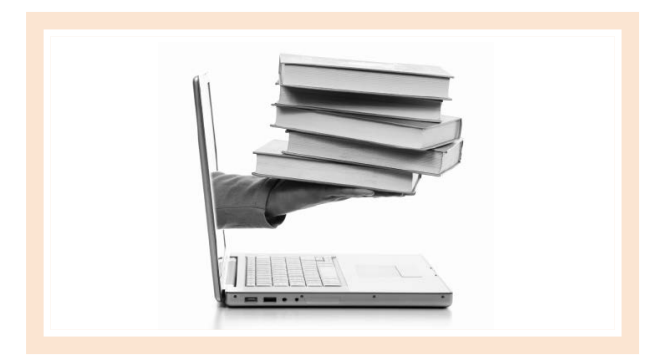

puedan originar y revisar hipótesis, diversas afirmaciones en contextos similares a los reales, potenciando la autorregulación del aprendizaje (Azevedo, 2005). El avance tecnológico permite ampliar el espectro de herramientas TIC a tablets y smartphones, que tienen la ventaja adicional de ofrecer la posibilidad de participar en redes y realizar trabajos en grupo, fomentando la creación de entornos de colaboración que establecen situaciones de corregulación entre diversos sujetos. Además, permiten a los alumnos representar su conocimiento sobre algún dominio para explorar el propio conocimiento.

Las TIC, además de facilitarnos información y comunicación, aportan «nuevas formas de pensar», potenciando la autonomía del estudiante en su proceso de aprendizaje y mejorando la eficacia de sus resultados. Pero para que estas herramientas puedan traernos todos los fundamentos que nos permitan progresar hacia un conocimiento más complejo y reflexivo, es necesaria una intervención educativa que permita un análisis claro de las restricciones y de las reglas implícitas que las propias tecnologías imponen. Es decir, no solo deben orientarse en los beneficios mencionados, sino también en cómo los individuos pueden ir construyendo sus metas, para lograrlas, y cómo pueden alcanzar su objetivo más importante en el proceso de aprendizaje (Coll y Monereo, 2008).

Por tanto, la adhesión metacognición y TIC conlleva una serie de cambios en cuanto al diseño, a la estructura y al funcionamiento de los métodos de aprendizaje y de las formas de proceder del docente.

\section{OBJETIVOS}

En el presente proyecto se distingue entre objetivos educativos y tecnológicos.

\subsection{Objetivos educativos}

- Diseñar actividades y tareas de aprendizaje orientados a conseguir una enseñanza de aprendizaje estratégico y autorregulado, comunicando de manera eficaz y clara. Estas actividades y tareas permitirán al alumno conocer su rendimiento de forma continua y mejorar su capacidad de «aprender a aprender». 
- Los alumnos, al recibir feedback inmediato de la realización de su tarea, deben llevar a la conciencia el aprendizaje obtenido, asi como sus errores y, consecuentemente, pensar en estrategias de reparación que puedan utilizar en la siguiente ocasión.

- Medir los niveles de satisfacción y motivación del alumnado ante la asignatura utilizando recursos tecnológicos.

\subsection{Objetivos tecnológicos}

- Utilizar de forma adecuada y eficaz herramientas tecnológicas, como vídeos, cuestionarios interactivos a los que se accede mediante códigos $Q R$, plataforma Moodle, dirigidas a la orientación, el seguimiento y la guía del alumno con el fin de conseguir un aprendizaje del contenido propuesto; en especial, herramientas que facilitan la gestión y el control del propio aprendizaje por parte del alumnado mediante la autoevaluación inmediata u on-line (elearning). Además, permitirá a los docentes obtener de manera instantánea informes detallados sobre el progreso de su propio alumnado por unidades temáticas de aprendizaje.
- Utilizar, aprovechando la motivación y ubicuidad que supone, un dispositivo móvil (tableta y/o teléfono móvil) con fines educativos, dándole un uso práctico responsable y diferente al ya conocido por los estudiantes (m-learning), sin depender del tiempo o la ubicación física, como sucede con otras herramientas TIC más tradicionales.

\section{METODOLOGÍA}

Las teorías metacognitivas sobre aprendizaje autorregulado dan una gran importancia a que exista un rápido feedback sobre los resultados del aprendizaje y a la contextualización del entorno del aprendizaje, esto es, a que haya una relación lo más estrecha posible entre los contenidos que se explican en las clases teóricas y las actividades prácticas que se realizan. Por ello, los bloques de contenidos teóricos se corresponden con una o varias clases prácticas, gracias a la coordinación previa que se da entre los dos profesores que imparten la materia (uno imparte teoría y el otro imparte prácticas) (véase tabla 1).

\section{Tabla 1. Relación entre teoría $(\mathrm{T})$ y práctica $(\mathrm{P})$ de la asignatura de Psicología Básica}

\begin{tabular}{|c|c|}
\hline Teoría & Práctica \\
\hline $\begin{array}{l}\text { Bloque 1. Introducción a la psicología } \\
\text { T1. ¿Qué es la psicología? Corrientes de psicología. Áreas de es- } \\
\text { pecialización y competencias del psicólogo en los equipos multipro- } \\
\text { fesionales. }\end{array}$ & $\begin{array}{l}\text { P1. La psicología es la ciencia que estudia la conducta y los procesos mentales. } \\
\text { Desde finales del siglo XIX han aparecido diversas escuelas con diferentes } \\
\text { perspectivas para estudiar a las personas. } \\
\text { Objetivo de la práctica: diferenciar entre las diferentes corrientes psicológicas y } \\
\text { sus aportaciones para la intervención social que aparecen en los textos que se } \\
\text { les facilitan. } \\
\text { P1. Ver guía didáctica tema } 1^{*} \text {. }\end{array}$ \\
\hline $\begin{array}{l}\text { Bloque 2. Biología y comportamiento } \\
\text { T2. Herencia y conducta. Funcionamiento del sistema nervioso. } \\
\text { Mente y cerebro. Alteraciones cerebrales. Disfunciones cognitivas } \\
\text { asociadas al cerebro. Cartografía cerebral. }\end{array}$ & $\begin{array}{l}\text { P2. La neuropsicología, disciplina que se dedica al conocimiento de la relación } \\
\text { existente entre cerebro y conducta, y sus alteraciones. Y la neuroimagen, técnica } \\
\text { que ha contribuido a comprender mejor el funcionamiento del cerebro. } \\
\text { Objetivo de la práctica: ser capaces de observar las diferencias entre los dos } \\
\text { tipos de cerebros, sano y patológico, y cómo nos pueden ayudar las técnicas de } \\
\text { neuroimagen en el diagnóstico y pronóstico de las patologías. } \\
\text { P2. Ver guía didáctica tema } 2^{*} \text {. }\end{array}$ \\
\hline
\end{tabular}


Tabla 1. Relación entre teoría $(T)$ y práctica $(P)$ de la asignatura de Psicología Básica (cont.)

\section{Teoria}

.......

\section{Bloque 3. Sueño, drogas y estados alterados}

T3. Conciencia, contenidos y funciones. Estados alterados de la conciencia. Ritmos biológicos. Ciclos del sueño. Alteraciones del sueño. Descripción básica de las drogas y sus efectos en el funcionamiento cognitivo.

\section{Bloque 4. Sensación}

T4. Principios básicos. Alteraciones y psicopatología.

\section{Bloque 5. Percepción y atención}

T5. Principios básicos. Tipos de atención. Procesos controlados y automáticos. Efecto Stroop.

\section{Bloque 6. Aprendizaje.}

T6. Categorías de aprendizaje. Condicionamiento clásico y operante y sus aplicaciones.

\section{Bloque 9. Memoria.}

T9. Naturaleza y mediciones. Clases de memoria. Representación del conocimiento. Teorías del olvido. Trastornos de la memoria. Reserva cognitiva y programas de mantenimiento.

\section{Bloque 11. Personalidad.}

T11. Naturaleza. Teorías del desarrollo. Controversia personasituación. Tipos de personalidad. Test.
P3. Sueño, estado fisiológico de autorregulación y reposo uniforme de todo organismo vivo. Importante un sueño reparador para un correcto descanso y adecuado funcionamiento de los procesos cognitivos. Pero existen ciertas alteraciones del sueño, en algunas personas, y diversas patologías que impiden que el sueño sea reparador.

Objetivo de la práctica: comprender la importancia de un sueño reparador y saber cómo conseguirlo

P3. Ver guía didáctica tema $3^{*}$.

P4. La sensación se refiere a los sentidos. Gracias a ellos tenemos sensaciones diferentes.

Es la captación de estimulación mediante células receptoras y conversión a impulsos nerviosos. Por tanto, debemos entender cómo recibimos los estímulos ambientales y cómo nos producen diferentes sensaciones.

Objetivo de la práctica: los alumnos deben experimentar diferentes sensaciones a través de los sentidos; además, deben identificarlas y definir, en cada una de ellas, los aspectos a los que responde el sistema sensorial.

P4. Ver guía didáctica tema $4^{*}$.

P5. Atención y percepción son procesos presentes en la mayoría de las tareas diarias que realizamos, gracias al sistema cognitivo.

Objetivo de la práctica: conocer los diferentes métodos existentes para la valoración de los diversos tipos de atención y poder interpretar los resultados obtenidos.

P5. Ver guía didáctica tema $5^{*}$

P6. A lo largo de la vida, cómo nos comportamos, cómo actuamos en el día a día es cuestión de aprendizaje, de educación. Existen diversas conductas que nos perjudican, a nivel personal y social, y deben ser reeducadas.

Objetivo de la práctica: elaboración, a nivel general, de un programa de reforzamiento conductual a partir de los diferentes aprendizajes estudiados.

P6. Ver guía didáctica tema $6^{*}$

P9. Memoria: capacidad cognitiva muy extensa y compleja que requiere de su conocimiento para promover una mejora de esta. Existen diferentes tipos de memoria y etapas, siendo además susceptibles de valoración mediante diversas pruebas y cuestionarios.

Objetivo de la práctica: observación de pruebas neuropsicológicas de valoración de la memoria y experimentación de una técnica de mejora de la memoria.

P9. Ver guía didáctica tema 9 *

P11. Personalidad, conjunto de rasgos y cualidades que constituyen la manera de ser de una persona y la diferencian de las demás. Cada persona tiene unos rasgos, ni buenos ni malos, pero una exaltación de algunos de ellos puede considerarse como no muy positivo cuando se ajusta a ciertos perfiles. Ejemplos claros de ello los tenemos en los permisos de obtención de armas.

Objetivo de la práctica: saber qué rasgos de personalidad se requieren para poder obtener un permiso de uso de armas de fuego (test EAE).

P11. Ver guía didáctica tema $11^{*}$. 
Tabla 1. Relación entre teoría (T) y práctica (P) de la asignatura de Psicología Básica (cont.)

\begin{tabular}{|c|c|}
\hline Teoría & Práctica \\
\hline \multicolumn{2}{|l|}{...$/ \ldots$} \\
\hline $\begin{array}{l}\text { Bloque 14. Psicología positiva. } \\
\text { T14. Naturaleza. El estado de bienestar. Áreas de estudio: fortalezas y } \\
\text { virtudes, flow y creatividad, resiliencia, inteligencia emocional, humor, } \\
\text { optimismo. }\end{array}$ & $\begin{array}{l}\text { P14. La psicología positiva es una de las ramas de la psicología más joven. Estudia } \\
\text { las emociones positivas, el desarrollo de las virtudes potenciando las propias } \\
\text { fortalezas y la búsqueda de la felicidad, bienestar, con el objetivo de conseguir una } \\
\text { mejor calidad de vida y bienestar. } \\
\text { Objetivo de la práctica: deben ser capaces de potenciar sus seis fortalezas más } \\
\text { significativas una vez realizado el cuestionario VIA-IS, así como elaborar, durante } \\
\text { la semana, un diario propio de gratitud. } \\
\text { P14. Ver guía didáctica tema } 14^{*} \text {. }\end{array}$ \\
\hline
\end{tabular}

(*) Para más información: https://cv4.ucm.es/moodle/course/view.php?id=63033.

Fuente: elaboración propia.

Durante el curso 2014-2015, para poder llevar a cabo una evaluación continua de las prácticas, se elaboraron cuestionarios web a los que se accedía mediante códigos $\mathrm{QR}$. De esta manera, y durante los últimos 10015 minutos de clase, cada alumno escaneaba el código y realizaba el cuestionario. En un breve espacio de tiempo, cada alumno recibía en su correo electrónico la calificación obtenida, así como sus aciertos y errores.

De un total de 14 prácticas (una por cada tema de teoría), 9 se diseñaron para evaluar el uso combinado de diferentes herramientas: role playing, dramatizaciones grupales, exposiciones orales, trabajos de investigación, etc. En la tabla 1 se muestran las prácticas que se evaluaron utilizando códigos QR.

\section{Antes del comienzo de las prácticas, se administraron tres instrumentos de evaluación (a los que se accedía a través de códigos QR) para conocer el punto de partida en el que se encontraba el alumnado}

\subsection{Muestra}

Los alumnos a los que se dirigió el presente proyecto fueron los matriculados en los grupos $A(A 1, A 2, A 3)$, $B(B 1, B 2, B 3)$ y $C(C 1, C 2, C 3)$ del primer curso del grado de Trabajo Social durante el curso académico 2014-2015, llegando a un total de 213 alumnos.

Todos disponían de dispositivo móvil y utilizaron la conexión wifi de la Universidad Complutense de Madrid.

\subsection{Materiales}

Dispositivo móvil, como teléfono o tablet con conexión a internet; proyector y pantalla del aula; ordenador del aula (con conexión a internet) y altavoces; manual de la asignatura (Puente, 2011); guía didáctica de la práctica a trabajar; artículos; lector de códigos QR; aplicación Movenote; cuestionarios Google Drive elaborados ad hoc; presentaciones PPT; vídeos de YouTube; páginas web; y plataforma Moodle.

\subsection{Instrumentos aplicados}

Antes del comienzo de las prácticas, se administraron tres instrumentos de evaluación (a los que se accedía a través de códigos QR) para conocer el punto de partida en el que se encontraba el alumnado: 


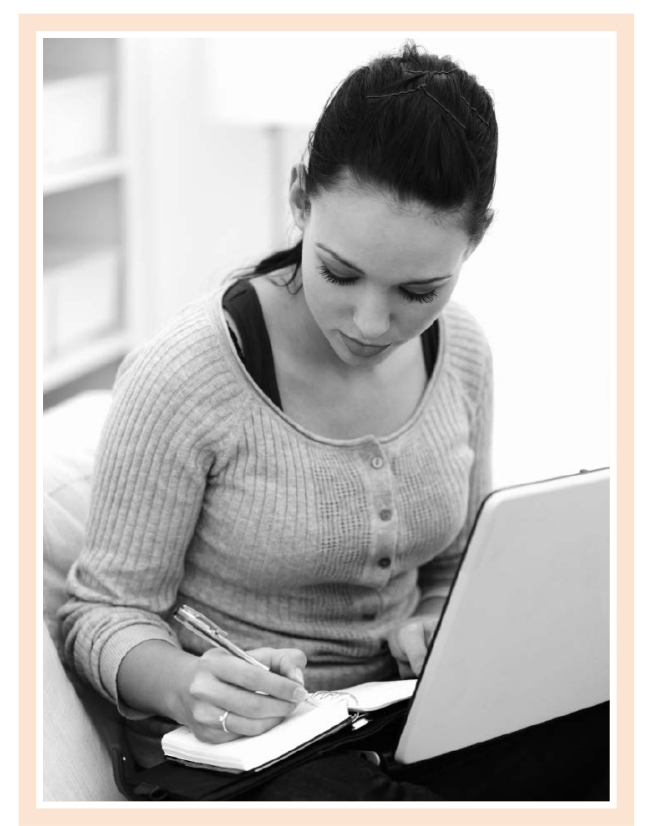

\section{- Cuestionario inicial.}

Cuestionario elaborado ad hoc para la investigación, donde únicamente se le pregunta al alumnado si conoce qué es el código $Q R$, si lo ha utilizado alguna vez y, en caso de respuesta afirmativa, que explique en qué situación lo ha hecho. Si lo conoce, también se le pregunta si lo considera útil para utilizarlo en el ámbito académico.

- Cuestionario MARSI (Mokhari y Reichard, 2002). Cuestionario compuesto por 30 ítems con respuesta Likert 5 puntos, que mide la conciencia metacognitiva que aplican los sujetos cuando leen. Este cuestionario se compone de tres subescalas: estrategias de lectura global, estrategias de solución de problemas y estrategias de apoyo a la lectura.

\section{- Cuestionario de uso de las TIC.}

Cuestionario elaborado ad hoc para la presente investigación compuesto por 28 ítems con respuesta Likert 5 puntos. En él se abordan tres grandes bloques de preguntas; uno relacionado con el uso de internet, otro relacionado con el uso del teléfono móvil y otro relacionado con el uso de las redes sociales.
Al finalizar las clases, además del cuestionario MARSI y el cuestionario TIC, también se les administró a los alumnos un cuestionario de satisfacción (de elaboración propia). Este cuestionario está compuesto por 18 ítems con respuesta Likert 5 puntos y una pregunta abierta («observaciones»), donde el estudiante puede añadir cualquier comentario relacionado con la asignatura que considere oportuno.

\subsection{Procedimiento}

\section{A) Sesión inicial}

Consistió en recoger los datos de los alumnos (cuestionario Inicial), así como en la administración de un cuestionario donde se registró la disponibilidad de recursos TIC (elaborado ad hoc) y una prueba de evaluación de estrategias metacognitivas (MARSI). A todas las pruebas se accedió a través de un código $\mathrm{QR}$ (pretest). La profesora proporcionó a los alumnos la información por escrito (véase anexo 1) y explicó, además, la metodología que se iba a seguir en las clases, indicándoles que todas las prácticas que estuvieran asociadas a cuestionarios interactivos se realizarían en el aula y que era requisito indispensable que llevaran consigo el teléfono móvil o la tableta, dispositivos en los que debían haberse descargado previamente una herramienta para leer códigos QR. La estructura de la clase tipo fue:

- Paso 1. Visualización de un vídeo de elaboración propia de 3 o 4 minutos de duración en formato MP3 donde la profesora explicó la tarea a realizar con apoyo de tres diapositivas insertadas en un PPT (aplicación Movenote).

- Paso 2. Acceso a un cuestionario interactivo sobre planificación de la tarea mediante un código QR que se expuso en clase.

- Paso 3. Realización de la tarea.

- Paso 4. Acceso a un cuestionario de evaluación de la tarea mediante otro código QR también expuesto en clase.

Además, cada práctica llevaba asociada su guía didáctica como un recurso bibliográfico más (véase https:// cv4.ucm.es/moodle/course/view.php?id=63033). 


\section{Tabla 2. Tarea que hay que realizar por cada práctica y recursos asociados}

\section{Práctica 1. Introducción a la psicología}

\begin{tabular}{l|l}
\hline Recursos bibliográficos & $\begin{array}{l}\text { Textos psicológicos, tabla de corrientes psicológicas extraída del manual de la asignatura (Puente, 2011). Capítulos } 1 \\
\text { y } 2 \text { del manual de la asignatura. } \\
\text { Védeo presentación práctica, cuestionarios web (planificación y evaluación), teléfono móvil o tableta. }\end{array}$ \\
Recursos TIC & $\begin{array}{l}\text { Análisis de un texto psicológico, identificando palabras clave, corrientes psicológicas, etc. } \\
\text { Tarea }\end{array}$ \\
Modalidad & Pequeño grupo e individual.
\end{tabular}

\section{Práctica 2. Biología y comportamiento}

\begin{tabular}{|l|l|}
\hline Recursos bibliográficos & $\begin{array}{l}\text { Capítulo } 4 \text { del manual de la asignatura (Puente, 2011). } \\
\text { Vídeo presentación práctica, PPT con fotografias de parejas de cerebros, cuestionarios web (planificación y } \\
\text { evaluación), teléfono móvil o tableta. }\end{array}$ \\
Tarea & $\begin{array}{l}\text { Observación en imágenes proyectadas de dos cerebros, uno sano y otro patológico, e Identificación de alteraciones } \\
\text { cerebrales. }\end{array}$ \\
Modalidad & Pequeño grupo e individual.
\end{tabular}

Práctica 3. Sueño, drogas y estados alterados

\begin{tabular}{|l|l|}
\hline Recursos bibliográficos & $\begin{array}{l}\text { Capítulo } 6 \text { del manual de la asignatura (Puente, 2011). } \\
\text { Recursos TIC }\end{array}$ \\
Vídeo presentación práctica, cuestionarios web (planificación y evaluación), teléfono móvil o tableta, páginas web \\
indicadas. \\
Marea & $\begin{array}{l}\text { Elaboración de un póster con el tema «Higiene del sueño» para un colectivo específico. } \\
\text { Pequeño grupo. }\end{array}$ \\
\hline
\end{tabular}

Práctica 4. Sensación

\begin{tabular}{|l|l|}
\hline Recursos bibliográficos & $\begin{array}{l}\text { Capítulo } 7 \text { del manual de la asignatura (Puente, 2011). } \\
\text { Vídeo presentación práctica, video YouTube, cuestionarios web (planificación y evaluación), teléfono móvil o tableta. }\end{array}$ \\
Recursos TIC & $\begin{array}{l}\text { Actividades de experimentación de sensaciones. Registro de diferentes aspectos de cada estímulo a los que responde } \\
\text { el sistema sensorial. }\end{array}$ \\
Modalidad & Individual y parejas.
\end{tabular}

Práctica 5. Percepción y atención

\begin{tabular}{|l|l|}
\hline Recursos bibliográficos & Matriz de Mesulam, PASAT, STROOP, CPT. Capítulo 9 del manual de la asignatura (Puente, 2011). \\
Recursos TIC & Vídeo presentación práctica, cuestionarios web (planificación y evaluación), teléfono móvil o tableta. \\
Tarea & Administración de diferentes test de atención e interpretación de resultados. \\
Modalidad & Gran grupo, pequeño grupo. \\
\hline
\end{tabular}

\section{Práctica 6. Aprendizaje}

\begin{tabular}{l|l}
\hline Recursos bibliográficos & Casos propuestos. Capítulos 10 y 11 del manual de la asignatura (Puente, 2011). \\
Recursos TIC & Video presentación práctica, cuestionarios web (planificación y evaluación), teléfono móvil o tableta. \\
Tarea & Elaboración de un programa de reforzamiento adecuado para el caso asignado. \\
Modalidad & Pequeño grupo.
\end{tabular}




\section{Tabla 2. Tarea que hay que realizar por cada práctica y recursos asociados (cont.)}

\begin{tabular}{|c|c|}
\hline \multicolumn{2}{|l|}{...$/ \ldots$} \\
\hline \multicolumn{2}{|l|}{ Práctica 9. Memoria } \\
\hline Recursos bibliográficos & $\begin{array}{l}\text { Subpruebas del test WAIS III (test de dígitos, test de letras y números), listados para aplicar método loci. Capítulo } 13 \\
\text { del manual de la asignatura (Puente, 2011). }\end{array}$ \\
\hline Recursos TIC & Vídeo presentación práctica, cuestionarios web (planificación y evaluación), teléfono móvil o tableta, páginas web. \\
\hline Tarea & Administración de pruebas de memoria, memorización de listados utilizando el método loci. \\
\hline Modalidad & Individual, pequeño grupo. \\
\hline \multicolumn{2}{|l|}{ Práctica 11. Personalidad } \\
\hline Recursos bibliográficos & Test EAE. Capítulo 20 del manual de la asignatura (Puente, 2011). \\
\hline Recursos TIC & $\begin{array}{l}\text { Vídeo presentación práctica, cuestionarios web (planificación y evaluación), teléfono móvil o tableta, página web, } \\
\text { presentación PPT de interpretación de resultados del test. }\end{array}$ \\
\hline Tarea & Realización del test EAE, interpretación de resultados. \\
\hline Modalidad & Individual. \\
\hline \multicolumn{2}{|c|}{ Práctica 14. Psicología positiva } \\
\hline Recursos bibliográficos & $\begin{array}{l}\text { Capítulo } 21 \text { del manual de la asignatura (Puente, 2011). Artículo: «Una aproximación al Trabajo Social desde la óptica } \\
\text { de la psicología positiva (virtudes y fortalezas)», Cuadernos de Trabajo Social, } 26 \text { (2), págs. 397-407; Ficha de «Diario } \\
\text { de gratitud». }\end{array}$ \\
\hline Recursos TIC & $\begin{array}{l}\text { Video presentación práctica, cuestionarios web (planificación y evaluación), teléfono móvil o tableta, páginas web. } \\
\text { Cuestionario VIA-IS. }\end{array}$ \\
\hline Tarea & Realización del cuestionario VIA-IS de fortalezas, análisis de las mismas y elaboración de un diario de gratitud. \\
\hline Modalidad & Individual \\
\hline
\end{tabular}

Fuente: elaboración propia.

\section{B) Sesión final}

El día de realización de la última sesión de prácticas, el alumnado volvió a realizar los cuestionarios de la primera sesión (postest) y un cuestionario de satisfacción sobre la metodología de las clases recibidas.

\section{ANÁLISIS DE RESULTADOS}

En un primer lugar, se pretende analizar si hay diferencias significativas entre las calificaciones del alumnado que ha cursado las prácticas sin el uso de códigos QR (271 alumnos en el curso 2013-2014) y los que sí lo han hecho (213 alumnos en el curso 2014-2015). Para ello, se ha realizado un ANOVA de un factor entre los alumnos del curso 20132014 y del curso 2014-2015. Durante el curso 2014-2015 se introdujeron códigos QR en la metodología docente y se quiere examinar la posible influencia de este recurso en el rendimiento de los alumnos. La media (SD) de las calificaciones de teoría, prácticas y final para los alumnos del curso 2013-2014 fue de 4,82 (1,06), 7,09 $(1,08)$ y $4,64(1,80)$, respectivamente. El promedio de calificaciones de teoría para el alumnado del curso 2014-2015 ha sido 5,60 (1,29), el de prácticas fue $7,06(1,11)$, y la media de la nota final, 5,92 $(1,78)$. La prueba de homogeneidad de varianzas nos permite afirmar que las varianzas son iguales para las calificaciones de prácticas $[F(1,482)=0,078 ; p=0,770]$, no así para las calificaciones de teoría $[F(1,482)$ $=4,336 ; p=0,038$ y para las calificaciones finales $(F=20,115 ; p<0,001)$. Se encuentra 
que las diferencias son estadísticamente significativas entre las puntuaciones de teoría, $F(1,482)=53,51$; con un $\mathrm{p}$-valor $<0,001$ y un tamaño del efecto $\eta 2=0,100$, al igual que entre las puntuaciones finales, $F(1,482)=59,91(\mathrm{p}$ $<0,001)$ y $\eta 2 p=0,111$. Luego, los grupos en los que se utilizaron códigos $Q R$ mostraron un mejor rendimiento académico que aquellos grupos en los que no se usaron.

\subsection{Análisis de las pruebas apli- cadas}

\section{A) Cuestionario de satisfacción}

A los alumnos del curso 2014-2015 se les aplicó una serie de pruebas antes de comenzar las clases prácticas y después de finalizarlas. Una de ellas fue el cuestionario de satisfacción. Los valores perdidos en los ítems 6, 7 , $9,11,12,14,15,17$ y 19 de este cuestionario fueron tratados utilizando la técnica de imputación múltiple. En concreto, se aplicó el método de especificaciones completamente condicionales con cinco iteraciones, siguiendo el modelo de regresión logística. Se ha escogido este método debido a la baja cantidad de valores perdidos de la muestra y a la ausencia de patrones claros sistemáticos de valores perdidos. También se comprobó la fiabilidad de este cuestionario. El valor de a de Cronbach de los datos originales y las imputaciones varía entre 0,904 y 0,905 , y su estandarización entre 0,917 y 0,919. La mejora de a que se consigue al descartar algunos de los ítems del cuestionario es mínima, invitando a mantener la configuración original de variables. Podemos afirmar que el cuestionario presenta buena consistencia interna. También estudiamos la dimensionalidad del cuestionario usando técnicas de análisis factorial. Las pruebas KMO y Barlett $\left(\mathrm{KMO}=0,921 ; \chi^{2}{ }_{153}=1724,070\right.$; $p<0,001)$ indican una buena adecuación de los datos al modelo de análisis factorial. El análisis paralelo indicó la presencia de un único factor que explicaba una alta proporción de varianza $>0,46$, de lo que se concluye que el cuestionario de satisfacción es fundamentalmente unidi- mensional. El ítem 19 de este cuestionario está reservado para que los alumnos recojan sus comentarios u observaciones. Se registraron un total de 59 observaciones. Estas observaciones se podrían categorizar como «comentarios generales positivos», "comentarios sobre metodología QR positivos», "comentarios generales negativos», «comentarios sobre metodología QR negativos» y «otros». Estas categorías serían excluyentes, no así la de «diferencias entre teoría y prácticas» (véase figura 2).

\section{Figura 2. Comentarios del ítem 19 del cuestionario de satisfacción}

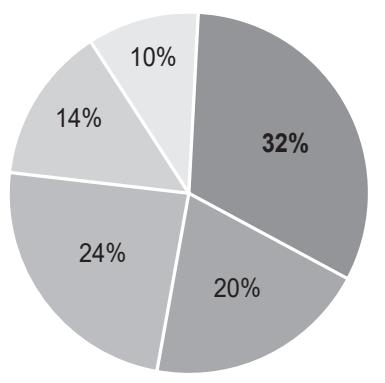

Comentarios generales positivos

Comentarios sobre metodología QR positivos

Comentarios generales negativos

Comentarios sobre metodología $Q R$ negativos

Otros

Fuente: elaboración propia.

Uno de los tópicos que se repiten con frecuencia en los comentarios generales positivos es lo entretenidas, amenas e interesantes que resultaron las clases de prácticas. Los comentarios positivos sobre la metodología QR se han centrado en su utilidad como complemento para el apartado de teoría. En los comentarios generales negativos, algunos alumnos apuntan la falta de tiempo como uno de los inconvenientes. La inclusión de una nueva herramienta en el ámbito docente no está exenta de contratiempos. Esto mismo es lo que registran algunos alumnos en sus comentarios: dificultades para conectarse a internet o problemas con los dispositivos móviles pueden/podrían llegar a entorpecer (incluso impedir) la realización de las prácticas. Otros comentarios, en cambio, se centran en las diferencias en la práctica docente entre los apartados de teoría y prácticas. 


\section{B) Cuestionario de uso de nuevas tecnologías (uso TIC)}

Además del cuestionario de satisfacción, se aplicó el cuestionario de uso de nuevas tecnologías (elaborado ad hoc). Al agrupar los ítems relacionados con el uso de internet, telefonía móvil y redes sociales, comparando las puntuaciones de las aplicaciones previa y posterior, se encontraron diferencias significativas en las dos primeras escalas. En la prueba de diferencia de medias entre las puntuaciones previas (pre) y posteriores (post) en la escala internet, $t_{212}=3,281$, con un p-valor $=$ 0,001 , y para las puntuaciones de la escala móvil, $t_{212}=$ 4,576 con $p<0,001$. Las diferencias entre las aplicaciones previa y posterior entre las puntuaciones totales del cuestionario también son significativas: $t_{212}=-3,856 ; p$ $<0,001$. Es especialmente relevante para este estudio comprobar si hay diferencias significativas en la utilidad percibida (en trabajos en grupo, búsqueda de información e investigación) y en las horas semanales (dedicadas a trabajos en grupo, búsqueda de información e investigación) en internet y móviles (véase tabla 3).

Las puntuaciones medias (SD) de utilidad de internet en las aplicaciones previa y posterior fueron 17 , $82(1,83)$ y $18,12(1,91)$, respectivamente. En cuanto al uso de internet, estas fueron $11,06(3,54)$ y 11,97 $(3,80)$. El promedio de puntuaciones de utilidad del teléfono móvil fue 15,27 $(3,53)$ para la primera aplica-

Tabla 3. Estadísticos descriptivos de las escalas del cuestionario de uso TIC

\begin{tabular}{|l|c|c|}
\hline \multicolumn{1}{|c|}{ Escalas } & Media & $\begin{array}{c}\text { Desviación } \\
\text { tipica }\end{array}$ \\
\hline Internet pre & 82,17 & 10,94 \\
Internet post & 84,20 & 11,74 \\
Móvil pre & 74,69 & 12,24 \\
Móvil post & 78,10 & 12,81 \\
Redes sociales pre & 53,72 & 11,72 \\
Redes sociales post & 54,69 & 12,14 \\
Total pre & 210,58 & 31,80 \\
Total post & 217,00 & 33,50 \\
\hline
\end{tabular}

$N=213$

Fuente: elaboración propia. ción y $15,86(3,54)$ para la segunda. Y con respecto al uso del teléfono móvil, la media de la aplicación previa fue $10,35(4,26)$ y $12,07(4,28)$ para la posterior. Se encuentran diferencias significativas en las medias de utilidad y uso tanto en internet como en móviles.

\section{C) Cuestionario MARSI de estrategias meta- cognitivas}

Al igual que con el cuestionario de satisfacción, se aplicó el cuestionario MARSI de estrategias metacognitivas al alumnado del curso 2014-2015 a principio y a final del año académico. Teniendo en cuenta las tres subescalas de las que está compuesto el cuestionario y la puntuación global del mismo, se compararon las puntuaciones de las aplicaciones previa y posterior en estas cuatro medidas (véase tabla 4). La media de las puntuaciones de la escala de estrategias de lectura global en la primera aplicación fue 48,10 $(6,20)$, y para la segunda aplicación, $51,68(6,21)$. Para la escala de solución de problemas en la primera aplicación fue $31,11(3,85)$, mientras que para la segunda aplicación esta fue $32,35(3,94)$. Para la escala de estrategias de apoyo lector, la aplicación previa tuvo de media 30,92 $(5,33)$, y la posterior, $34,00(4,97)$. El promedio de la puntuación total de la primera aplicación fue 110,13 $(12,99)$, mientras que para la segunda fue 118,03 $(13,55)$. Se encontraron diferencias estadísticamente significativas para las cuatro medidas (véase tabla 4).

Tabla 4. Contraste de medias para las medidas del cuestionario MARSI

\begin{tabular}{|l|c|c|}
\multicolumn{1}{|c|}{ Medidas } & t & Sig. \\
\hline $\begin{array}{l}\text { Escala de estrategias globales de } \\
\text { lectura pre y post }\end{array}$ & $-9,268$ & $<, 001$ \\
$\begin{array}{l}\text { Estrategias de solución de } \\
\text { problemas pre y post }\end{array}$ & $-4,384$ & $<, 001$ \\
$\begin{array}{l}\text { Escala de estrategias de apoyo en la } \\
\text { lectura pre y post }\end{array}$ & $-8,579$ & $<, 001$ \\
Puntuación total pre y post & $-9,051$ & $<, 001$ \\
\hline
\end{tabular}

$N=213$

Fuente: elaboración propia. 
Se han encontrado correlaciones significativas entre las medidas del cuestionario MARSI (escalas y puntuación total) y la puntuación total del cuestionario de satisfacción. Estas correlaciones son significativas tanto para las aplicaciones pre, como para las aplicaciones post del cuestionario MARSI, aunque estas correlaciones sean más altas en las aplicaciones posteriores.

Al estudiar las medidas del MARSI con las escalas del cuestionario de uso TIC también se encuentran correlaciones estadísticamente significativas. Al estudiar las puntuaciones de estas dos pruebas destacan las correlaciones significativas entre las escalas de internet, móvil y uso de móvil con todas las medidas de MARSI (véase tabla 5).

Como se aprecia en la tabla 6 , las correlaciones son mayores en las medias posteriores (salvo en uso de móvil). Incluso se encuentran nuevas correlaciones significativas con las medidas de MARSI: a la escala de internet, móvil y uso de móvil se suman utilidad de internet $y$ utilidad de móvil.

Igual ocurre con el cuestionario de satisfacción. Las correlaciones son mayores en las medias posteriores (salvo en uso de móvil) (véase tabla 7).

Para concluir, hay que resaltar también que se han encontrado correlaciones significativas entre las calificaciones de prácticas y la puntuación total de satisfacción (véase tabla 8).
Tabla 5. Correlaciones entre medidas previas del MARSI y escalas del cuestionario de uso TIC

\begin{tabular}{|l|c|c|c|c|c|c|}
\multicolumn{1}{|c|}{ Medidas } & $\begin{array}{c}\text { Internet } \\
\text { pre }\end{array}$ & $\begin{array}{c}\text { Móvil } \\
\text { pre }\end{array}$ & $\begin{array}{c}\text { Utilidad } \\
\text { internet pre }\end{array}$ & $\begin{array}{c}\text { Uso } \\
\text { internet pre }\end{array}$ & $\begin{array}{c}\text { Utilidad } \\
\text { móvil pre }\end{array}$ & $\begin{array}{c}\text { Uso } \\
\text { móvil pre }\end{array}$ \\
\hline $\begin{array}{l}\text { Estrategias globales de } \\
\text { lectura pre }\end{array}$ & $0,248^{* *}$ & $0,224^{* *}$ & 0,126 & 0,104 & 0,033 & $0,219^{* *}$ \\
$\begin{array}{l}\text { Estrategias de solución de } \\
\text { problemas pre }\end{array}$ & $0,171^{*}$ & $0,271^{* *}$ & 0,042 & 0,070 & 0,109 & $0,267^{* *}$ \\
$\begin{array}{l}\text { Estrategias de apoyo en la } \\
\text { lectura pre }\end{array}$ & $0,180^{* *}$ & $0,225^{* *}$ & $-0,041$ & $0,143^{*}$ & 0,132 & $0,237^{* *}$ \\
Puntuación total pre & $0,243^{* *}$ & $0,280^{* *}$ & 0,056 & 0,129 & 0,102 & $0,281^{* *}$ \\
\hline
\end{tabular}

$N=213 ;{ }^{*} p<, 05 ; * * p<0,01$

Fuente: elaboración propia.

Tabla 6. Correlaciones entre medidas posteriores del MARSI y escalas del cuestionario de uso TIC

\begin{tabular}{|c|c|c|c|c|c|c|}
\hline Medidas & $\begin{array}{l}\text { Internet } \\
\text { post }\end{array}$ & $\begin{array}{l}\text { Móvil } \\
\text { post }\end{array}$ & $\begin{array}{l}\text { Utilidad } \\
\text { internet post }\end{array}$ & $\begin{array}{c}\text { Uso } \\
\text { internet post }\end{array}$ & $\begin{array}{l}\text { Utilidad } \\
\text { móvil post }\end{array}$ & $\begin{array}{l}\text { Uso } \\
\text { móvil post }\end{array}$ \\
\hline $\begin{array}{l}\text { Estrategias globales de } \\
\text { lectura post }\end{array}$ & $0,319^{* *}$ & $0,276^{* *}$ & $0,316^{* *}$ & $0,147^{*}$ & $0,221^{* *}$ & $0,167^{*}$ \\
\hline $\begin{array}{l}\text { Estrategias de solución de } \\
\text { problemas post }\end{array}$ & $0,249^{* *}$ & $0,295^{* *}$ & $0,249^{* *}$ & 0,053 & $0,265^{\star *}$ & $0,139^{*}$ \\
\hline $\begin{array}{l}\text { Estrategias de apoyo en la } \\
\text { lectura post }\end{array}$ & $0,271^{* *}$ & $0,285^{* *}$ & $0,269^{* *}$ & $0,161^{*}$ & $0,272^{* *}$ & $0,171^{*}$ \\
\hline Puntuación total post & $0,318^{* *}$ & $0,317^{* *}$ & $0,316^{* *}$ & $0,142^{*}$ & $0,278^{* *}$ & $0,180^{* *}$ \\
\hline
\end{tabular}

$N=213 ;{ }^{*} p<, 05 ;{ }^{* *} p<0,01$

Fuente: elaboración propia.

Tabla 7. Correlaciones entre medidas MARSI y puntuación total del cuestionario de satisfacción

\begin{tabular}{|l|c|c|}
\hline \multicolumn{1}{|c|}{ Medidas } & $\mathrm{t}$ & Sig. \\
\hline Escala de estrategias globales de lectura pre y puntuación total de & 0,178 &, 009 \\
satisfacción & & \\
Estrategias de solución de problemas pre y puntuación total de satisfacción & 0,163 &, 017 \\
Escala de estrategias de apoyo en la lectura pre y puntuación total de & 0,140 &, 041 \\
satisfacción & & \\
Puntuación total pre y puntuación total de satisfacción & 0,191 &, 005 \\
Escala de estrategias globales de lectura post y puntuación total de satisfacción & 0,326 & $<, 001$ \\
Estrategias de solución de problemas post y puntuación total de satisfacción & 0,306 & $<, 001$ \\
Escala de estrategias de apoyo en la lectura post y puntuación total de & 0,297 & $<, 001$ \\
satisfacción & & \\
Puntuación total post y puntuación total de satisfacción & 0,348 & $<, 001$ \\
\hline
\end{tabular}

$N=213$

Fuente: elaboración propia. 
Tabla 8. Correlaciones entre las calificaciones y las puntuaciones totales del cuestionario de satisfacción

\begin{tabular}{|l|c|c|c|c|}
\hline \multicolumn{1}{|c|}{ Medidas } & $\begin{array}{c}\text { Calificación } \\
\text { de teoria }\end{array}$ & $\begin{array}{c}\text { Calificación } \\
\text { de prácticas }\end{array}$ & $\begin{array}{c}\text { Calificación } \\
\text { final }\end{array}$ & $\begin{array}{c}\text { Puntuación } \\
\text { total de } \\
\text { satisfacción }\end{array}$ \\
\hline Calificación de teoría & 1 & & & \\
Calificación de prácticas & $0,298^{* *}$ & 1 & & \\
Calificación final & $0,907^{* *}$ & $0,447^{* *}$ & 1 & \\
$\begin{array}{l}\text { Puntuación total de } \\
\text { satisfacción }\end{array}$ & $-0,031$ & $0,193^{* *}$ & 0,061 & 1 \\
\hline
\end{tabular}

$N=213 ;{ }^{*} p<, 05 ; * * p<0,01$

Fuente: elaboración propia.

\author{
Una información inmediata \\ como la que proporcionan los \\ códigos QR ayuda a hacer \\ interpretaciones adecuadas, \\ permitiendo adaptar las \\ creencias previas e incorporar \\ estrategias de afrontamiento \\ más efectivas
}

\section{CONCLUSIONES}

La presente investigación ha mostrado que la implementación de la evaluación continua y el uso de las TIC mediante el uso de los códigos QR tiene un efecto positivo sobre el rendimiento académico y la motivación de los alumnos, incrementa sus niveles de satisfacción (los alumnos indicaron que el uso de códigos QR les fue de gran utilidad para lograr superar la asignatura) y, lo que es más importante, moviliza recursos metacognitivos relacionados con la autorregulación, lo que explicaría las mejoras en la adquisición de conocimientos y habilidades mostradas por los estudiantes.

\begin{abstract}
Al estudiar las puntuaciones en metacognición y aprendizaje autorregulado de MARSI y el cuestionario TIC de la aplicación posterior encontramos que surgen correlaciones significativas entre las medidas de MARSI y las escalas de utilidad de internet y de móvil. Con utilidad se refiere al uso que se le puede dar a estas nuevas tecnologías en el ámbito educativo (trabajos en grupo, búsqueda de información e investigación). Es a este nivel donde podríamos encontrar los beneficios de usar los códigos $Q R$. Aunque las nuevas tecnologías estén muy presentes en nuestro día a día, no terminan de hacerse hueco en el ámbito educativo. Muestra de ello son los comentarios de algunos alumnos sobre las diferencias entre el apartado de teoría y prácticas.
\end{abstract}

De acuerdo con la teoría cognitiva social de Bandura, los individuos controlan sus pensamientos, sentimientos y acciones a través de un sistema de autorregulación. De este sistema depende el ejercicio de control y acción personal. Ahora bien, para que pueda operar de forma adaptativa es preciso modular las creencias sobre las propias capacidades en relación con los resultados obtenidos. Una información inmediata como la que proporcionan los códigos QR ayuda a hacer interpretaciones adecuadas, permitiendo adaptar las creencias previas e incorporar estrategias de afrontamiento más efectivas. La autoevaluación inmediata que proporcionan los códigos QR influye, además, sobre las percepciones de autoeficacia, modulando el pensamiento y la afectividad. Una alta autoeficacia ayuda a crear sentimientos de serenidad al abordar las tareas y actividades complejas. Por lo tanto, las creencias de autoeficacia se convierten en factores determinantes y son fuertes predictores del nivel de logro que los individuos finalmente puedan alcanzar.

Se hace manifiesta la necesidad de estos proyectos e incorporar las nuevas tecnologías al ámbito educativo e ir actualizándose. El propósito no sería tanto hacer que con las nuevas tecnologías el alumnado consiga aprobar sus asignaturas, sino que aprendan a usarlas y a sacarles provecho en el proceso de enseñanza-aprendizaje.

Se esperaba una mejoría básicamente en la satisfacción del alumnado respecto al uso de estos recursos tecnológicos y su motivación hacia el aprendizaje, así como en el uso de estrategias metacognitivas para alcanzar un aprendizaje autónomo. Los análisis arrojan resultados muy positivos donde el entorno de aprendizaje y el clima de aula se ven favorecidos. Esto nos hace reflexionar sobre la importancia de un verdadero cambio metodológico en el paradigma educativo universitario actual. 


\section{BIBLIOGRAFÍA}

Azevedo, R. [2005]: «Using a hypermedia as a metacognitive tool for enhancing student learning? The role of self-regulated learning», Educational Psychologist, 40 (4), págs. 199-209.

Brown, A. L. [1980]: «Metacognitive development and reading», en R. J. Spiro, B. C. Bruce y W. F. Brewer (eds.), Theoretical issues in reading comprehension, Hillsdale (NJ): LEA.

[1987]: «Metacognition, executive control, self-regulation and other more mysterious mechanisms», en $\mathrm{F}$. Weinert y R. Kluwe (eds.), Metacognition, motivation and understanding. Hillsdale (NJ): LEA.

Burón, J. [1996]: Enseñar a aprender: introducción a la metacognición, Bilbao: Ediciones Mensajero.

Coll, C. y Monereo, C. [2008]: Psicología de la educación virtual, Madrid: Morata.

Delgado, A. M.; Borge, R.; García, J.; Oliver, R. y Salomón, L. [2005]: Competencias y diseño de la evaluación continua y final en el Espacio Europeo de Educación Superior, Programa de Estudios y Análisis, Universitat Oberta de Catalunya y Universidad Pompeu Fabra, Ministerio de Educación y Ciencia.

Flavell, J. H. [1971]: «First discussant's comments. What is memory development the development of?», Human Development, 14, págs. 272-278.

[1976]: «Metacognitive aspects of problem solving», en B. Resnick (ed.), The nature of intelligence, Hillsdale (NJ): Erlbaum.

[1981]: «Cognitive monitoring», en W. P. Dickson (ed.), Children's oral comunication skills, New York: Academic Press.

[1987]: «Speculations about the nature and development of metacognition», en F. Weinert y R. Kluwe (eds.), Metacognition, motivation and understanding, Hillsdale (NJ): LEA.

Flavell, J. H. y Wellman, H. M. [1977]: «Metamemory», en R. V. Kail Jr. y J. W Hagen (eds.), Perspectives on the development of memory and cognition, Hillsdale (NJ): LEA, págs. 3-33.

Jiménez, V. [2004]: Metacognición y comprensión de la lectura: evaluación de los componentes estratégicos (procesos y variables) mediante la elaboración de una escala de conciencia lectora (ESCOLA), Madrid: Universidad Complutense de Madrid.

Jiménez, V.; Puente, A.; Alvarado, J. M. y Arrebillaga, L. [2009]: «La medición de las estrategias metacognitivas mediante la escala de conciencia lectora ESCOLA», Revista de Investigación Psicoeducativa, 7 (2), págs. 185-205.

Llopis, C.; Jiménez, V. y Benítez, F. [2015]: «Programa de entrenamiento en estrategias metacognitivas y uso responsable de herramientas TIC», en R. Santiago, S. Trabaldo, M. Kamijo y A. Fernández (eds.), Mobile learning: nuevas realidades en el aula, Digital Text.

Mateos, M. M. [2001]: Metacognición y educación, Buenos Aires: Aique.

Mokhari, K. y Reichard, C. A. [2002]: «Assessing student's metacognitive awareness of reading strategies», Journal of Educational Psychology, 94 (2), págs. 249-259.

Müller, W. [2009]: «Metacognición y TIC: una combinación que permite la construcción de escenarios posibles en educación», Interactic, 12 (2), págs. 1-12.

Pintrich, P. R. y Groot, E. V. de [1990]: «Motivational and self-regulated learning components of classroom academic performance», Journal of Educational Psychology, 82 (1), págs. 33-40.

Pozo, J. I. y Monereo, C. [2009]: «La nueva cultura del aprendizaje universitario o por qué cambiar nuestras formas de enseñar y aprender», en J. I. Pozo y M. P. Pérez Echevarría (eds.), Psicología del aprendizaje universitario: la formación en competencias, Madrid: Morata, págs. 9-28.

Puente, A. [2011]: Psicología contemporánea básica y aplicada, Madrid: Pirámide.

Ríos, P. [1991]: «Metacognición y comprensión de la lectura», en A. Puente (ed.), Comprensión de la lectura y acción docente, Madrid: Fundación Germán Sánchez Ruipérez, págs. 275-298.

Schraw, G. [1998]: «Promoting general metacognitive awareness», Instructional Science, 26 (1-2), págs. 113125.

Suárez, N.; Núñez, J. C.; Fernández, E.; Cueli, M. y García, T. [2012]: «Efecto diferencial de un programa de intervención sobre los enfoques de aprendizaje en educación superior: ¿modalidad presencial o virtual?», III Jornadas Internacionales de Campus Virtuales, Universidad de Oviedo.

Zapata, M. [2012]:«Evaluación de la calidad en entornos sociales de aprendizaje», III Jornadas Internacionales de Campus Virtuales, Universidad de Murcia. 


\section{ANEXOS}

\section{Práctica Psicología Básica (curso 2014-2015)}

\section{Estimado alumno:}

Hoy comienzas las prácticas de Psicología Básica. Para un correcto funcionamiento de las mismas te ruego máxima puntualidad y traer cuanto antes la ficha de subgrupo.

En la mayor parte de las sesiones vas a poder evaluar tu propio aprendizaje gracias al uso de códigos QR. Para poder acceder a la lectura de un código QR (QR codes) es imprescindible que tengas instalado en el terminal móvil (teléfono 0 tableta con acceso a internet) un lector adecuado para poder realizar la lectura a través de la cámara de tu dispositivo.

Existen muchos lectores de códigos QR de descarga gratuita. Como recomendación:

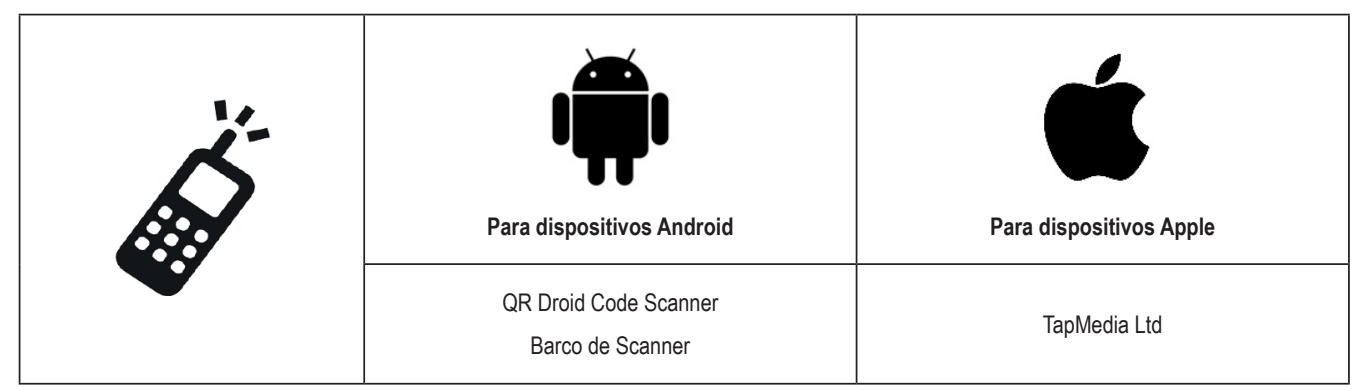

Además, en cada sesión puedes traer el manual de la asignatura.

Para comenzar es obligatorio que realices los siguientes cuestionarios:

\begin{tabular}{|c|c|c|}
\hline Cuestionario inicial & TIC & MARSI \\
\hline 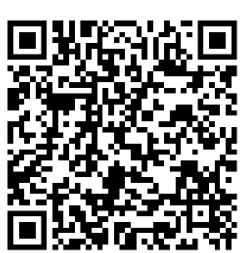 & 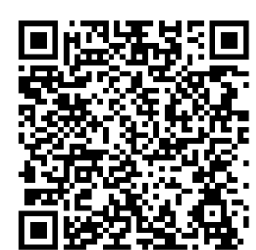 & 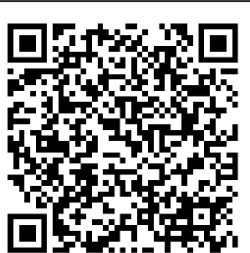 \\
\hline
\end{tabular}




\section{Alumnos trabajando con códigos QR}
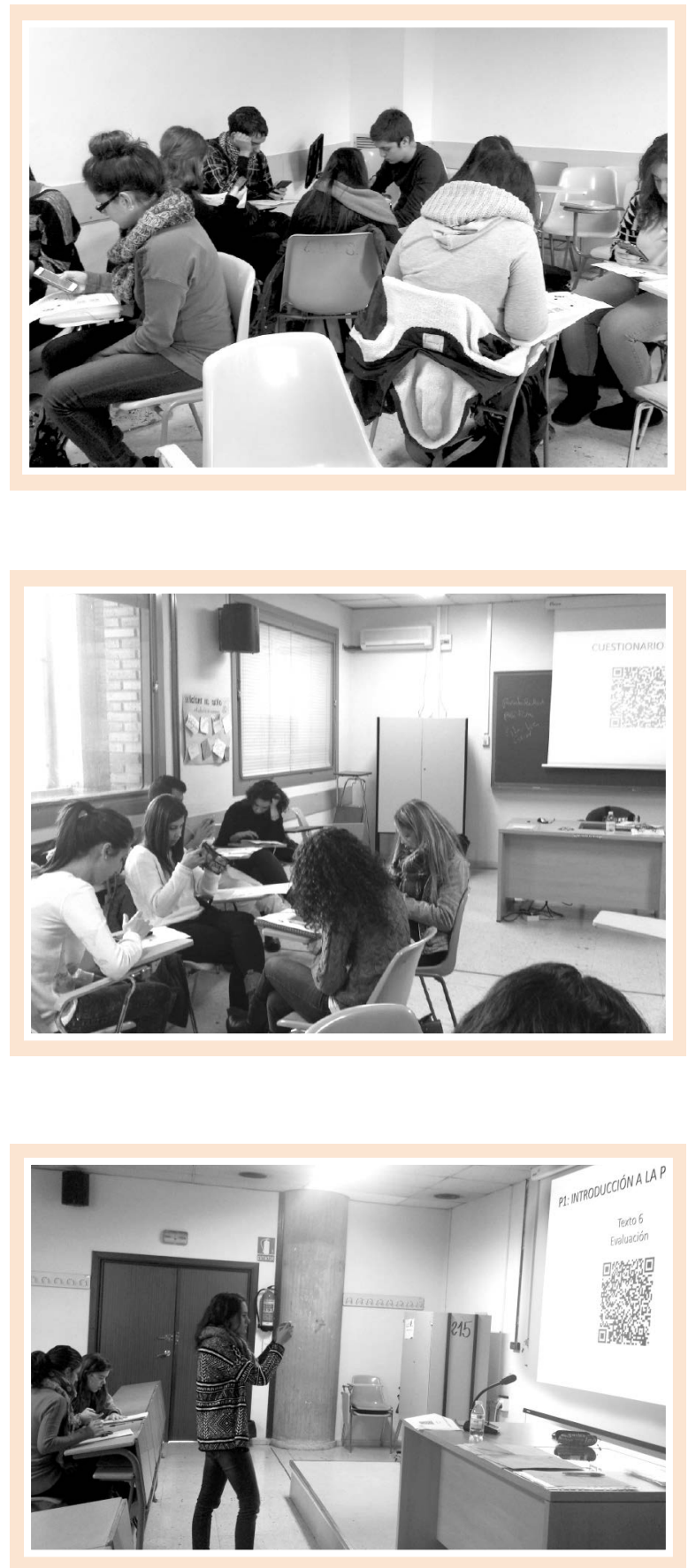
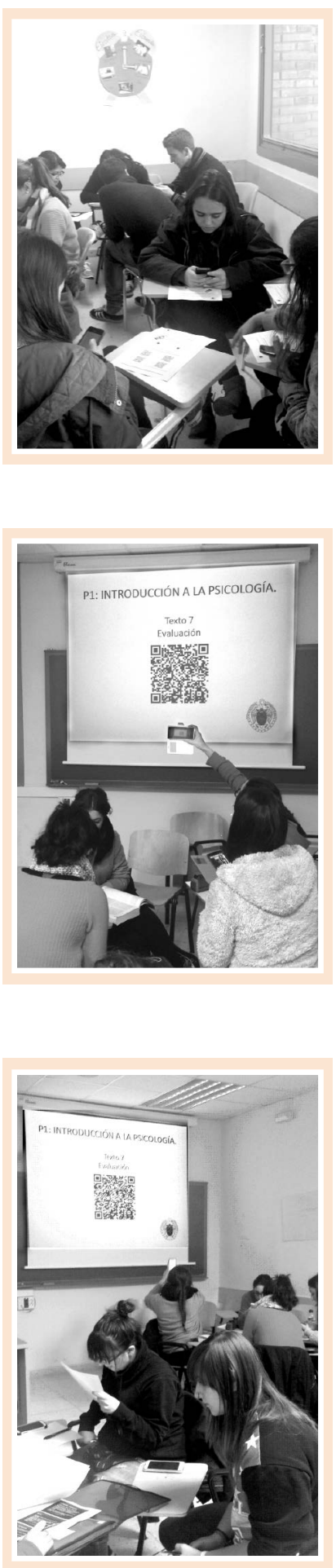
La Másteres oficiales que ofrece la UDIMA tienen como señas de identidad la cercanía del profesor al alumno y la mejor aplicación posible de las TIC a la formación. La UDIMA es una institución de enseñanza pensada y diseñada para las personas que trabajan y para quienes por motivos de horarios, movilidad, distancia geográfica o de conciliación familiar demandan una universidad abierta y flexible. Estos Másteres oficiales [60 créditos ECTS] se inician en octubre y febrero de cada año y su duración normal es de 12 meses.

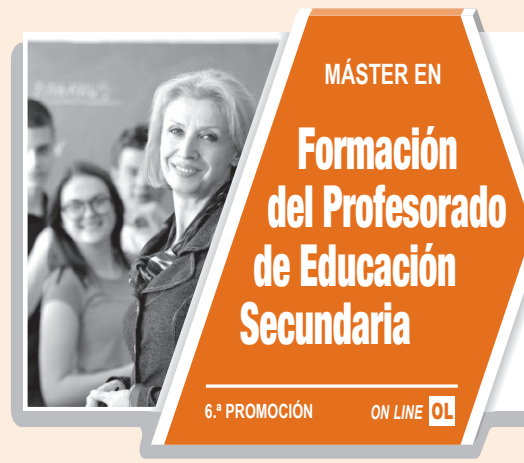

DIRIGIDO A: La Orden ECI/3858/2007 establece que los alumnos que deseen acceder a este máster, además de los requisitos generales, deben cumplir: acreditación del dominio de las competencias relativas a la especialización que se desee cursar y dominio de una lengua extranjera equivalente al nivel B1 del Marco Común Europeo de Referencia para las lenguas.

OBJETIVOS: Adquirir las competencias necesarias para poder ejercer la profesión docente en Secundaria, Bachillerato, Formación Profesional y enseñanzas de idiomas.
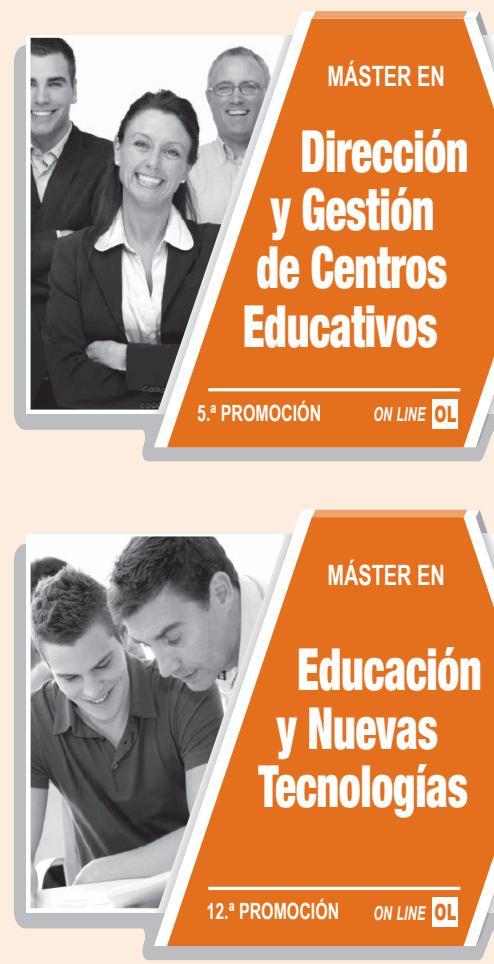

DIRIGIDO A: Titulados universitarios que quieran especializarse en el ámbito de la dirección y gestión de centros educativos. Este programa formativo no exige una experiencia previa, sino que pretende proporcionar una formación completa para la dirección y gestión de los centros.

OBJETIVOS: Capacitar a los profesionales para el ejercicio eficaz de la función directiva y de gestión de centros educativos. Para ello, el máster pretende dotar al alumno de las competencias y herramientas necesarias para el trabajo en un equipo multidisciplinar de profesionales, así como para promover la calidad y la innovación dentro del sistema educativo. 